Provided for non-commercial research and education use. Not for reproduction, distribution or commercial use.

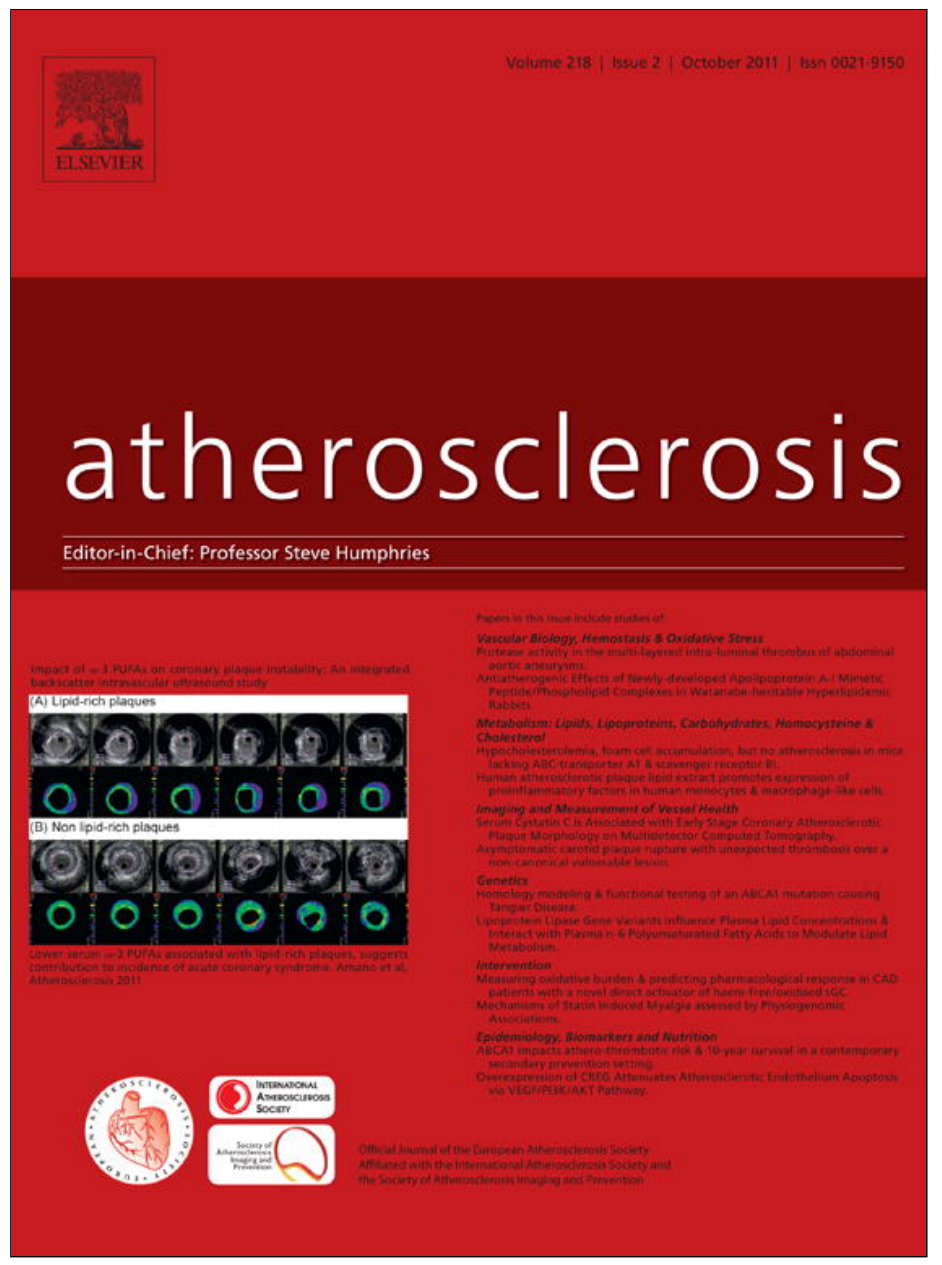

This article appeared in a journal published by Elsevier. The attached copy is furnished to the author for internal non-commercial research and education use, including for instruction at the authors institution and sharing with colleagues.

Other uses, including reproduction and distribution, or selling or licensing copies, or posting to personal, institutional or third party websites are prohibited.

In most cases authors are permitted to post their version of the article (e.g. in Word or Tex form) to their personal website or institutional repository. Authors requiring further information regarding Elsevier's archiving and manuscript policies are encouraged to visit:

http://www.elsevier.com/copyright 


\title{
Asymptomatic carotid plaque rupture with unexpected thrombosis over a non-canonical vulnerable lesion
}

\author{
Alessandro Mauriello ${ }^{\mathrm{a}, *}$, Francesca Servadei $^{\mathrm{a}}$, Giuseppe Sangiorgi $^{\mathrm{b}}$, Lucia Anemona $^{\mathrm{a}}$, Erica Giacobbi $^{\mathrm{a}}$, \\ Doriana Liotti ${ }^{\mathrm{a}}$, Luigi Giusto Spagnoli ${ }^{\mathrm{a}}$ \\ a Anatomic Pathology, University of Rome Tor Vergata, Italy \\ ${ }^{\mathrm{b}}$ Department of Cardiology, University of Rome Tor Vergata, Italy
}

\section{A R T I C L E I N F O}

\section{Article history}

Received 11 May 2011

Received in revised form 23 June 2011

Accepted 28 June 2011

Available online 12 July 2011

\section{Keywords:}

Carotid

Thrombosis

Asymptomatics

Pathology

\begin{abstract}
A B S T R A C T
Objective: Several studies have demonstrated that carotid plaque rupture and thrombosis represent the most important factors correlated with the onset of acute cerebrovascular symptoms. Nevertheless, ruptured thrombotic plaques have been described also in asymptomatic patients. What still needs to be clarified is why a plaque rupture leads either to an acute ischemic syndrome or, in a minor group of patients, remains asymptomatic. The purpose of this study was to systematically compare the histologic features of thrombotic plaques both in asymptomatic and symptomatic patients in order to identify specific findings that could explain the peculiar clinical behavior that characterizes each of the clinical settings.

Methods: A total of 157 thrombotic plaques from 60 asymptomatic patients and 97 with major stroke who consecutively underwent CEA were serially sectioned and studied by histology.

Results: A minute cap disruption very frequently characterizes thrombotic plaques of asymptomatic patients and it was always smaller than large ulcers observed in thrombotic symptomatic plaques $(651 \pm 687 \mu \mathrm{m}$ vs. $4150 \pm 3526, p=0.001)$. In asymptomatics this typical feature was associated with fewer inflammatory cells $(20.1 \pm 8.8$ vs. $33.9 \pm 26.1$ cells $\times$ hpf, $p=0.001)$, smaller lipidic-necrotic core $(33.9 \% \pm 2.9 \%$ vs. $42.0 \% \pm 2.4 \% ; p=0.04)$ and larger calcification $(16.2 \pm 12.8 \%$ vs. $8.1 \pm 12.2 \%, p=0.02)$. Symptomatic patients with thrombotic plaques showed higher incidence of metabolic syndrome $(p=0.002)$ and moderate-high Framingham risk scores $(p=0.001)$ comparing to asymptomatic individuals.

Conclusion: The transformation from a stable to a vulnerable plaque is a gradual process in the natural history of the disease and plaque rupture is an event not necessarily occurring at a late phase but also at earlier one. In this case, the rupture will be most likely smaller and clinically asymptomatic.
\end{abstract}

(c) 2011 Elsevier Ireland Ltd. All rights reserved.

\section{Introduction}

The importance of carotid atherosclerosis and related thromboembolic occlusion of a large artery tributary of the brain in the pathogenesis of ischemia and cerebral infarction has been acknowledged for many years [1-4]. Although the grade of stenosis due to a plaque encroaching the lumen constitutes the major criterion to identify subgroups of patients at high risk of stroke [5,6], results

\footnotetext{
* Corresponding author at: Cattedra di Anatomia ed Istologia Patologica, Dipartimento di Biopatologia e Diagnostica per Immagini, Universita' di Roma "Tor Vergata", Via Montpellier 1, 00133 Roma, Italy. Tel.: +39 06 2023751; fax: +3906 20902209

E-mail address: alessandro.mauriello@uniroma2.it (A. Mauriello).
}

from several studies suggest that plaque rupture and thrombosis represent the most important factor correlated with the onset of symptoms [1,3]. In a previous study, we observed a ruptured and thrombotic plaque in $74 \%$ of patients with ipsilateral stroke and in $100 \%$ of patients treated within 2 months from symptoms onset [1]. This strong correlation between the plaque thrombosis and an acute cerebrovascular disease has been confirmed by other morphologic and imaging-based studies $[7,8]$. Thus, these data suggest that 2 types of carotid artery disease can occur: one stable form, unlikely to produce symptomatic embolization or carotid occlusion and a second, unstable form at high risk of embolization or carotid occlusion, the latter is not necessarily being more stenotic.

Many efforts have been recently made using non-invasive techniques [8-12] for identifying plaques at high risk of disruption leading to thrombosis, generally defined as "vulnerable plaques" 
$[13,14]$. These latter lesions are characterized by a large necrotic core with an overlying thin cap rich in inflammatory cells. Unlike the stable plaque which shows a chronic inflammatory infiltrate, both vulnerable and ruptured plaques are characterized by a chronic "active" inflammation [15].

Ruptured plaques have been also described in asymptomatic patients. While ruptured symptomatic plaques have been widely examined, only few data are available on asymptomatic ruptures. What still needs to be clarified is why a plaque rupture can lead to an acute ischemic syndrome or, alternatively, in a minor group of patients, remain asymptomatic.

The aim of this study was to systematically compare the histologic features of thrombotic plaques both in asymptomatic and symptomatic patients in order to identify specific findings that could explain the peculiar clinical behavior that characterizes each of the clinical settings.

\section{Materials and methods}

\subsection{Selection of cases}

From the 486 consecutive carotid endarterectomies present in our database - The Interinstitutional Carotid Tissue Bank [1] - and collected from 2006 to 2010, a total of 157 thrombotic plaques from 60 asymptomatic patients and 97 with major stroke formed the basis of the study. A routine pre-operative evaluation in all patients included: clinical assessment of risk factor profile, a cerebral CT scan study, a selective angiographic examination of extra and intracranial carotid arteries and their branches.

Major stroke was defined as a clinical syndrome characterized by rapidly developing focal or at times global symptoms without significant clinical improvement within 7 days in the distribution of symptomatic carotid artery, not hemorrhagic and with no cause other than vascular origin, assessed by brain CT study as a cortical or deep white matter or basal ganglia lesion bigger than $1 \mathrm{~cm}$ [1]. Asymptomatic patients had never suffered from neurological symptoms and had no cerebral lesions assessed by CT.

The time interval between symptom onset and CEA in stroke patients was from 1 to 6 months. Aspirin was administered to all patients prior to surgery.

The study was approved by the institutional review boards and all patients gave consent for entry into the study. Histopathologic examination was performed in the pathology core lab of the University of Rome Tor Vergata by three different pathologists (AM, FS, LGS) blinded to the clinical data.

\subsection{Histologic sampling and light microscopy}

Carotid plaques were removed en bloc during surgery to entirely preserve plaque structure. Surgical samples were fixed for $24 \mathrm{~h}$ in $10 \%$ buffered formalin immediately upon removal. The specimens were sectioned transversally every $5 \mathrm{~mm}$ and paraffin embedded. Haematoxylin-eosin and Movat pentachrome stains were performed for morphological study. Each segment was sequentially numbered to reconstruct the entire plaque length. For each plaque, three to ten sections were examined according to the plaque length (mean 5 sections per specimen).

The presence of acute thrombosis, cap rupture or erosion, minimum cap thickness, cross-sectional area of the lipidic-necrotic core, intraplaque hemorrhage and calcification were evaluated per each plaque. The inflammatory infiltrate was also studied using the following monoclonal antibodies: CD68 (anti human macrophages; Dakopatts, Denmark), CD163 (anti M2 macrophages, Novocastra Leica, United Kingdom) and CD3 (anti-human T cell; Dakopatts) monoclonal antibodies.
An ultrastructural study was also performed in selected cases. Tissue samples were postfixed in $1 \%$ phosphate-buffered osmium and embedded in epoxy resin (Epon 812). Ultrathin sections were examined by means of Hitachi H-7100FA electron microscope.

\subsection{Atherosclerotic plaque types and histologic definitions}

Plaques were classified, according to the modified American Heart Association (AHA) atherosclerosis classification [14] into thrombotic and non-thrombotic.

Thrombotic plaques were characterized by the presence of an acute thrombus associated to plaque rupture, erosion or calcified nodule. Plaque rupture was defined by an area of fibrous cap disruption whereby the overlying acute thrombus was in continuity with the underlying necrotic core [14]. Ruptures were further classified into two subgroups according to their dimension: (a) $\leq 1 \mathrm{~mm}$ (also called fissure) and (b) $>1 \mathrm{~mm}$ (called ulceration). Plaque erosion was identified when serial sectioning of a thrombosed arterial segment failed to reveal fibrous cap rupture [14]. Calcified nodule referred to a lesion with luminal thrombus associated with an eruptive, dense area of calcium and underlying calcific plate [14]. Some plaques were classified as thrombotically active (TAP) if an acute thrombus was present over an organized or an organizing thrombus [1].

Non-thrombotic plaques included vulnerable plaques or thin cap fibroatheromata, fibrous cap atheromata, fibrocalcific lesions and healed plaque ruptures.

As vulnerability criteria for carotid plaque the presence of a thin fibrous cap $(<165 \mu \mathrm{m})$, heavily infiltrated by macrophages ( $>25$ cells per high power field $-\mathrm{hpf}$, done at a magnification of $40 \times$ using a test grid with an area of $0.22 \mathrm{~mm}^{2}$ ) associated to a large lipidic core $(30-40 \%$ of plaque area) were considered $[13,16]$.

\subsection{Risk factors}

The following risk factors were evaluated: hypertension, diabetes mellitus, cigarette smoking (former smokers who had stopped smoking for $<5$ years were considered as smokers and patients who had not smoked for $>5$ years were considered as nonsmokers), hypercholesterolemia, elevated triglyceridemia (TG), low HDL-cholesterol (HDL-C), abdominal obesity (patients with a waist circumference $\geq 102 \mathrm{~cm}$ in men or $\geq 88 \mathrm{~cm}$ in women [5]). In addition, we evaluated the presence of metabolic syndrome and the Framingham risk score [17]. The metabolic syndrome was defined, according to the scientific statement from the AHA and the National Heart, Lung, and Blood Institute (NHLBI) [18], if any 3 of the following 5 abnormalities were present: (1) elevated waist circumference, (2) elevated TG, (3) elevated blood pressure (BP) ( $\geq 130 \mathrm{mmHg}$ systolic BP or $\geq 85 \mathrm{mmHg}$ diastolic BP or drug treatment), (4) elevated fasting glucose ( $>100 \mathrm{mg} / \mathrm{dL}$ or drug treatment), and (5) low HDL-C. The Framingham risk score [17] was calculated utilizing an algorithm which includes age, gender, smoking status, presence of diabetes as dichotomous parameters, as well as total cholesterol, HDL-C, diastolic and systolic BP using the categories defined in the Framingham equation.

\subsection{Statistical analysis}

Data were analyzed by SPSS 13.0 (Statistical Package for the Social Sciences) software. Continuous and categorical variables are expressed as mean \pm SD and as frequency values and proportions, respectively. Pearson's chi-square test was utilized to assess possible differences of dichotomous variables between plaques of the various groups examined. The means of normally distributed data were compared with Student's $t$ test. In the other cases the groups were compared with Mann-Whitney's $U$ test. Multivariate analy- 
sis using stepwise logistic regression (using the "enter" method for variable selection) was utilized to identify independent risk factors which significantly correlate with plaque thrombosis. Receiver operating characteristic curves (ROC; for ulcer size, number of inflammatory cells, lipidic-necrotic core \% size, and calcification $\%$ size) with corresponding area under the curve (AUC) have been evaluated to describe the optimal cut-offs for distinction between asymptomatic and symptomatic plaques. In addition, the AUCs of ROCs from different plaque features were compared according to Vergara et al. [19]. The ROC curves provided several cutoff points to show the trade-off between sensitivity and specificity at different cutoff values. Sensitivity and specificity values were calculated with confidence interval analysis according to the Wilson method. A probability value $<0.05$ was considered statistically significant.

\section{Results}

\subsection{Thrombotic plaque characteristics}

Out of 157 thrombotic plaques, 95 (38 asymptomatic patients and 57 with stroke) showed an acute thrombosis, while in the remaining 62 plaques an organizing thrombus (TAP) was found (Table 1). The incidence of thrombosis was significantly higher in patients affected by stroke, compared to asymptomatic patients $(p=0.02)$. The grade of luminal narrowing of the thrombotic segment was significantly lower in asymptomatic than in symptomatic patients $(58.1 \pm 11.9 \%$ vs. $68.0 \pm 12.8 \%, p=0.01$, Table 1$)$. In about $90 \%$ of asymptomatic patients thrombosis was found in segments with $<70 \%$ stenosis.

In 24 out of 38 asymptomatic patients with acute thrombosis (63.1\%) a ruptured plaque was found. It is noteworthy that asymptomatic plaque ruptures were significantly smaller than those found in stroke patients $(651 \pm 687 \mu \mathrm{m}$ vs. $4150 \pm 3526$ respectively, $p=0.001$ ) (Fig. 1). In fact, in 20 out of 24 cases the rupture was characterized by only a small fissure $(<1 \mathrm{~mm})$.

Moreover $10.5 \%$ of asymptomatic patients (4/38) showed a plaque erosion associated with the presence of scattered macrophages and numerous mast cells (Fig. 2). In all these cases a calcification underneath the eroded area was observed.

Finally, in $26.3 \%$ of asymptomatic patients with thrombotic plaques (10/38) a calcified nodule was present (Fig. 2).

The location of acute thrombotic plaques was in the common carotid in 11 cases (11.6\%, 5 asymptomatic and 6 symptomatic), at level of bifurcation in 59 (62.1\%; 22 asymptomatic and 37 symptomatic) and in the remaining 25 cases in the internal carotid (26.3\%, 11 asymptomatic and 14 symptomatic), with no significative differences $(p=0.67)$. Moreover, no statistical differences were observed in the incidence of various types of plaques rupture between patients treated and non-treated with statins $(p=0.78)$.

\subsection{Features of vulnerability}

The thickness of fibrous cap near the rupture site in asymptomatic plaques was about twice that of plaques of stroke patients $(183.2 \pm 67.1$ vs. $84.4 \pm 38.6, p=0.001)$ and showed a mild macrophagic infiltrate, mainly of M2 phenotype (Fig. 1D). The inflammatory infiltrate in asymptomatic plaques was about half of that observed in symptomatic ones (20.1 \pm 7.2 vs. $33.9 \pm 14.1$ cells $\times$ hpf, $p=0.001$ ).

Asymptomatic ruptured plaques showed also a significantly higher extension of calcification as compared to stroke patients. In fact, the relative area occupied by calcium deposits in asymptomatic ruptured plaques was $16.2 \pm 12.8 \%$, while in symptomatic thrombotic lesions it was $8.1 \pm 12.2 \%(p=0.02)$. Similarly, in asymptomatic thrombotic plaques the relative area of lipidic-necrotic core was smaller than that of symptomatic ones $(33.9 \% \pm 2.9 \%$ vs. $42.0 \% \pm 2.4 \% ; p=0.04$ ).

Intraplaque hemorrhage is another finding that we frequently observed both in non-ruptured and in thrombotic plaques of asymptomatic patients. We cannot establish if the hemorrhage is a consequence of the blood flowing from the lumen through the cap rupture or if it represents, on the contrary, the cause of the cap rupture. This latter hypothesis is supported by the observation of many small and thin-walled vessels within the fibrous cap. Moreover, the ultrastructural examination showed that these microvessels lack interendothelial junctions, explaining the fragility of these vascular channels (Fig. 1E and F).

We also constructed ROC curves by plotting sensitivity (the proportion of true-positive results) vs. 1-specificity (the proportion of false-positive results) and determined the corresponding AUC to show the ability of some plaque morphological features to discriminate symptomatic from asymptomatic rupture. Both minimum cap thickness ( $\mathrm{AUC}=0.90,95 \% \mathrm{CI} 0.61-1.00$ ) and calcification $(\mathrm{AUC}=0.70,95 \% \mathrm{Cl} 0.59-0.81$ ) showed a moderate diagnostic accuracy, AUC being in the interval 0.7-0.9 [20]. The ROC curves also provided several cutoff points to show the tradeoff between sensitivity and specificity at different cutoff values. For minimum cap thickness, the optimal threshold was $122 \mu \mathrm{m}$ corresponding to $86 \%$ sensitivity and $82 \%$ specificity, whereas for calcification area the optimal threshold was $7.5 \%$ with $65 \%$ sensitivity and $72 \%$ specificity. Regarding other plaque characteristics the optimal cutoff was $715 \mu \mathrm{m}$ for ulcer size (sensitivity $=44 \%$, specificity $=86 \%$ ), 27 cells $\times$ hpf for inflammatory infiltrate (sensitivity $=21 \%$, specificity $=56 \%$ ) and $42.5 \%$ for the relative area of lipidic-necrotic core (sensitivity $=50 \%$, specificity $=41 \%$ ).

In symptomatic plaques no significative correlation was observed between plaque morphologic features and time from symptom onset and endarterectomy.

\subsection{Correlation with risk factors}

The univariate analysis showed that among the single risk factors only hypertension and low HDL-C were more frequently observed in symptomatic than in asymptomatic patients $(p=0.04$ and $p=0.02$, respectively) (Table 2 ). Moreover, symptomatic patients with thrombotic plaques showed higher incidence of metabolic syndrome comparing to asymptomatic individuals ( $47.4 \%$ vs. $15.8 \%$ respectively, $p=0.002$ ). Similarly, moderate-high Framingham risk scores were more common in symptomatic patients $(68.4 \%)$, while in the asymptomatic group low-medium scores were prevalent $(p=0.001)$. Multivariate analysis showed similar results, confirming that metabolic syndrome and Framingham risk score were independent predictive factors for the symptomatic carotid thrombosis.

\section{Discussion}

Our results demonstrate that asymptomatic ruptures and thrombosis frequently occur in the carotid district. Asymptomatic thrombotic plaques differ from symptomatic ones for the presence of a small disruption of a thicker cap, fewer inflammatory cells mainly represented by M2 type macrophages, smaller lipidic-necrotic core and larger calcification. These features are not completely consistent with the morphological definition of a vulnerable plaque, mainly as regarding cap thickness and amount of inflammatory infiltrate (Table 1 ).

The present study suggests that carotid plaques could show several levels of vulnerability correlated to different histological features. The acquisition of vulnerability is a gradual process and plaque rupture is an event not necessarily occurring at the end 
Table 1

Morphological findings of thrombotic plaques.

\begin{tabular}{|c|c|c|c|}
\hline & \multicolumn{2}{|l|}{ Thrombotic plaques } & \multirow[t]{2}{*}{$p$} \\
\hline & Asymptomatic (60 patients) & Stroke (97 patients) & \\
\hline \multicolumn{4}{|l|}{ Plaque types } \\
\hline (A) Acute thrombosis & $38(63.3)$ & $57(58.8)$ & 0.02 \\
\hline Fissure $(<1 \mathrm{~mm})$ & $20(33.3)$ & $5(5.1)$ & \\
\hline Ulceration (>1 mm) & $4(6.7)$ & $42(43.3)$ & \\
\hline Erosion & $4(6.7)$ & $3(3.1)$ & \\
\hline Calcified nodule & $10(16.6)$ & $7(7.2)$ & \\
\hline (B) Organizing thrombosis & $22(36.7)$ & $40(41.2)$ & \\
\hline Rupture dimension $(\mu \mathrm{m} \pm \mathrm{SD})$ & $651 \pm 687$ & $4150 \pm 3526$ & 0.001 \\
\hline Stenosis of thrombotic segment $(\%+\mathrm{SD})$ & $58.1 \pm 11.9$ & $68.0 \pm 12.8$ & 0.01 \\
\hline \multicolumn{4}{|l|}{ Features of vulnerability } \\
\hline Cap thickness $(\mu \mathrm{m} \pm \mathrm{SD})$ & $183.2 \pm 67.1$ & $84.4 \pm 38.6$ & 0.001 \\
\hline Cap macrophagic infiltrate (no. of cells $/ \mathrm{hpf} \pm \mathrm{SD}$ ) & $20.1 \pm 7.2$ & $33.9 \pm 14.1$ & 0.001 \\
\hline Lipidic-necrotic core (area $\% \pm \mathrm{SD})$ & $33.9 \pm 2.9$ & $42.0 \pm 2.4$ & 0.04 \\
\hline Calcification (area $\% \pm \mathrm{SD}$ ) & $16.2 \pm 12.8$ & $8.1 \pm 12.2$ & 0.02 \\
\hline
\end{tabular}

of the natural history of the disease but also in an earlier phase. The accepted criteria for the definition of a carotid vulnerable plaque are the presence of a thin fibrous cap $(<165 \mu \mathrm{m})$, heavily infiltrated by macrophages ( $>25$ cells per hpf) associated to a large lipidic core (30-40\% of plaque area) $[13,16]$. This type plaque is at high risk of wide symptomatic ruptures and thrombosis. Our study demonstrates that there are "non canonical" vulnerable plaques characterized by mild inflammation, thicker fibrous cap, that will undergo small asymptomatic ruptures and repair. Prospective studies using imaging methods could determine if a plaque type represents an earlier vs. later stage of vulnerability. Today it is impossible to say whether each patient will progress through different stages of vulnerability. It is possible that asymptomatic patients will always be asymptomatic, and that symptomatic patients never had asymptomatic ruptures.

In asymptomatic lesions the presence of few macrophagic cells, mainly of M2 subtype, as compared to symptomatic plaques, suggests that not only a smaller infiltration, but also a different pattern of inflammation correlate with asymptomatic plaque rupture. It has been established that monocytes, in response to different cytokine pattern and immunological microenvironment, can differentiate in two major subpopulations, referred to as M1 and M2 macrophages. M1 macrophages are considered to promote atherosclerosis development and plaque rupture, secreting cytokines and lytic enzymes involved in the thinning of fibrous cap, while M2 macrophages are expected to inhibit plaque growth and to promote reparative processes, mediating plaque stability $[21,22]$. Since in asymptomatic plaque ruptures we observed an intense M2 response, it could be hypothesized that in these lesions a reparative response rapidly occurs. Nevertheless we cannot establish if M2 macrophages were already present in the plaque before the rupture occurred or if they were attracted following cap disruption as a consequence of thrombosis and hemorrhage. In fact, it has been recently demonstrated that M2 macrophages are able to bind and clear haemoglobin-haptoglobin complexes from the vessel wall using the scavenger receptor CD163 [23].

Also in asymptomatic erosions we observed a modest inflammatory infiltrate. Nevertheless, several mast cells were found (Fig. 2E and F). It has been demonstrated that subendothelial mast cells, by inducing apoptosis of endothelial cells [24], may induce detachment of endothelium from the cap thus determining plaque erosion.

It has been widely accepted that major coronary and cerebrovascular risk factors increase vascular inflammation, thus mediating plaque instability [16]. Although most risk factors have an independent effect, there may be important interactions between them, amplifying their action. Our observation that patients with symptomatic thrombotic plaques, characterized by a greater inflammatory activity, had a significantly higher Framingham risk score as compared to that of asymptomatic individuals, seems to confirm this hypothesis. Similarly, it is not surprising that symptomatic patients with highly inflamed plaques and wide cap ruptures and thrombosis tend to be more frequently affected by metabolic syndrome. Metabolic syndrome is a common and complex disorder combining obesity, dyslipidemia, hypertension and

Table 2

Risk factors and thrombotic plaques.

\begin{tabular}{|c|c|c|c|c|}
\hline \multirow[t]{2}{*}{ Risk factors } & \multicolumn{2}{|l|}{ Thrombotic plaque } & \multirow[t]{2}{*}{ Univariate analysis $(p)$} & \multirow[t]{2}{*}{ Multivariate analysis $(p)$} \\
\hline & Asymptomatic (38 patients) & Stroke (57 patients) & & \\
\hline Age, mean $( \pm \mathrm{SD})$ & $70.2 \pm 7.5$ & $70.1 \pm 7.8$ & 0.97 & 0.80 \\
\hline Sex, $N(\%)$ & $30(78,9)$ & $47(82.5)$ & 0.93 & 0.62 \\
\hline \multicolumn{5}{|l|}{ Male } \\
\hline Female & $8(21.1)$ & $10(17.5)$ & & \\
\hline Hypertension, $N(\%)$ & $23(60.5)$ & $47(82.5)$ & 0.04 & 0.17 \\
\hline Diabetes, $N(\%)$ & $5(13.2)$ & $13(22.8)$ & 0.27 & 0.76 \\
\hline Cigarette smoking, $N(\%)$ & $21(55.3)$ & $40(70.2)$ & 0.21 & 0.47 \\
\hline Hypercholesterolemia, $N(\%)$ & $23(60.5)$ & $40(70.2)$ & 0.46 & 0.82 \\
\hline Low HDL-C, $N(\%)$ & $13(34.2)$ & $35(61.4)$ & 0.02 & 0.12 \\
\hline Hypertriglyceridemia, $N(\%)$ & $13(34.2)$ & $29(50.9)$ & 0.15 & 0.56 \\
\hline Abdominal obesity, $N(\%)$ & $4(10.5)$ & $9(15.8)$ & 0.57 & 0.86 \\
\hline Metabolic syndrome, $N(\%)$ & $6(15.8)$ & $27(47.4)$ & 0.002 & 0.005 \\
\hline \multicolumn{5}{|l|}{ Framingham risk score, $N(\%)$} \\
\hline Low-medium & $26(68.4)$ & $18(31.6)$ & 0.001 & 0.001 \\
\hline Moderate-high & $12(31.6)$ & $39(68.4)$ & & \\
\hline
\end{tabular}

In bold statistically significant differences are reported. 

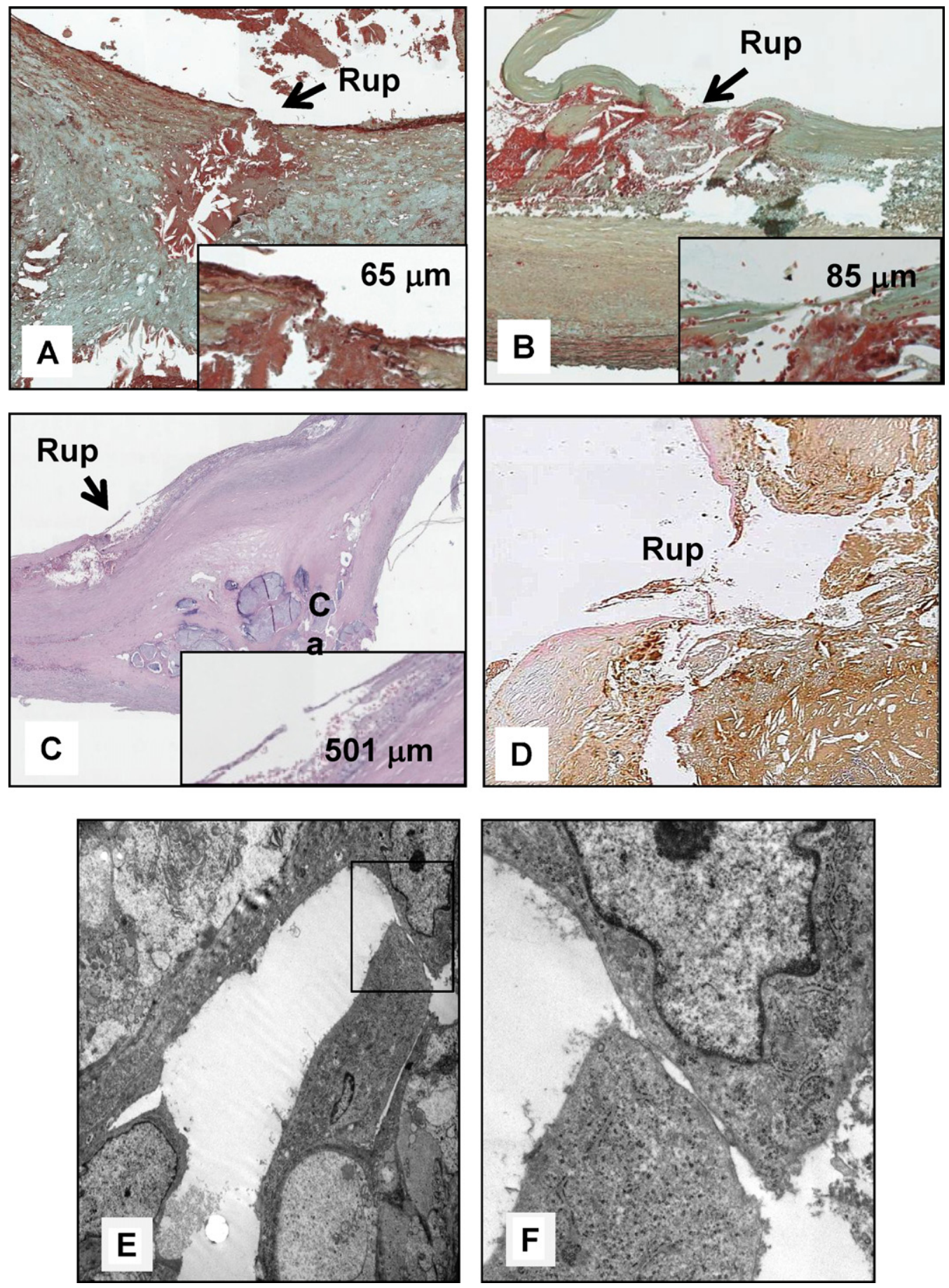

Fig. 1. Histopathology of an asymptomatic carotid plaque rupture and thrombosis. Panels A-C: micrographs showing different histologic plaque rupture samples from asymptomatic patients submitted to surgical carotid endarterectomy. Rupture sites (Rup) are characterized by a small disruption (arrow) of the thin fibrous cap with overlying acute thrombus in continuity with necrotic core debris. In the inset a magnification of rupture site is reported with its dimension. In the panel $\mathrm{C}$ a large calcification is present under the small cap rupture (A-B: Movat pentachrome stains, $4 \times$; C: Haematoxylin-eosin stain, $2 \times$ ). Panel D: the fibrous cap near the rupture site showed a mild macrophagic infiltrate, mainly of M2 phenotype, positive to CD163 antibody (10×). Panel E: an intramural hemorrhage associated to the presence of many small and thin-walled vessels within the fibrous cap was frequently observed in plaques of asymptomatic patients. The ultrastructural examination showed that these microvessels lack interendothelial junctions, explaining the fragility of these vascular channels. A magnification of (E) is reported in the Panel F.

insulin resistance. Hyperinsulinemia and impaired glycaemic control were associated with an increase of in vivo LDL oxidation and inflammation $[25,26]$. Dyslipidemia, associated with high levels of triglycerides and low HDL concentrations, contributes to a proinflammatory state $[27,28]$. Moreover, increasing experimental evidences support the hypothesis that also hypertension induces an increment of oxidative stress in the arterial wall, favoring therefore the recruitment of the inflammatory cells $[16,29,30]$.
It is noteworthy that $26.3 \%$ of acute thrombosis in asymptomatic patients has been observed in correspondence with a calcified nodule. This kind of lesion has been described in coronary circulation [14] and it is rarely observed in carotids. Subendothelial calcified nodules in asymptomatic patients are usually localized in the context of thin cap plaques and mildly stenotic segments. Both these conditions, according to Laplace law, make these plaques highly susceptible to rupture [13,31]. Moreover, it has to be emphasized 

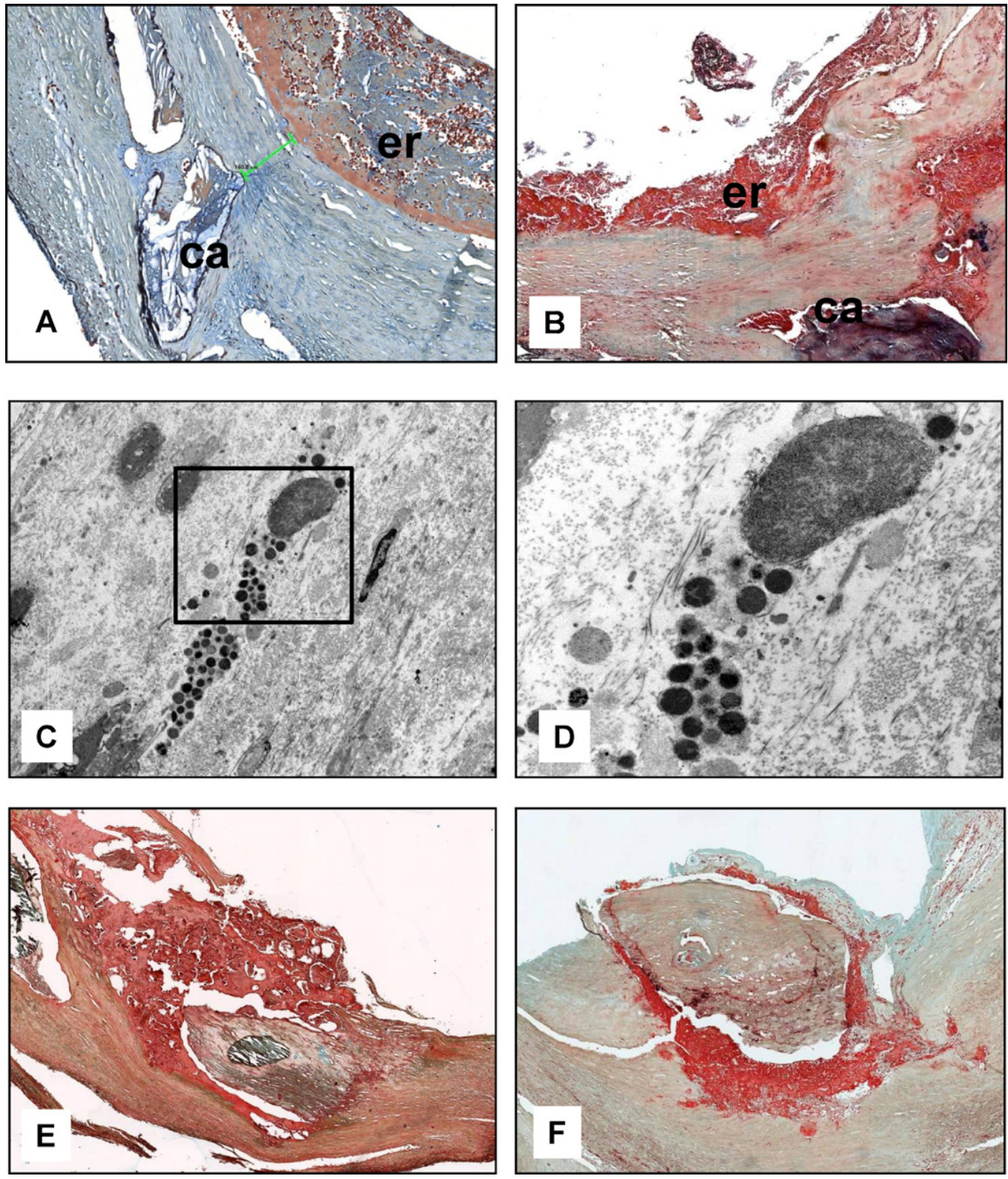

Fig. 2. Histopathology of asymptomatic carotid plaque erosions and calcified nodule. Panels A and B: micrographs showed two plaque erosions (er) associated to the presence of broad calcific plates (ca) (Movat pentachrome stains, $4 \times$ ). Panel C: transmission electron micrograph of a mast cell in the subepithelial space under the plaque erosion. A magnification of $(C)$ is reported in the Panel D. Panels E and F: micrographs showed three asymptomatic carotid plaques constituted by a luminal thrombus associated with an eruptive, dense area of calcium and underlying calcific plate (Movat pentachrome stains, $4 \times$ ).

that these calcified nodules, protruding into the vascular lumen, can induce flow disturbances, increase the stiffness and reduce the elasticity of the vessel wall [32].

In our cases, broad calcific plates have been also observed in the other subtypes of asymptomatic thrombotic plaques, such as rupture (Fig. 1C) and erosion (Fig. 2A and B). This finding is only apparently in contradiction with previous data reported in the literature showing that in the carotid the calcium deposition is associated with stable lesions $[33,34]$. In fact, in our cases symptomatic thrombotic plaques have little calcifications, while wide calcific plates occur in the small asymptomatic plaque ruptures.In conclusion, our results demonstrate that two types of plaque rupture can occur in the carotid, probably related to different pathogenetic mechanisms. When plaque rupture is associated with the onset of an acute cerebrovascular event, a wide cap disruption can be found in the context of a lesion showing the classic features of vulnerable plaque (high active inflammation, thin fibrous cap). On the contrary, when small-sized plaque ruptures occur, those are characterized by mild inflammation, thicker fibrous cap, broad calcification and mural hemorrhage, and are most likely correlated with an increased parietal stress. This rupture is likely to undergo repair and organization. The organization of these small asymptomatic ruptures could represent a frequent mechanism of plaque growth, as suggested by the fact that in $20 \%$ of asymptomatic patients a healed lesion has been observed.

\section{Financial support}

This study was supported by grant from MURST (MURST did not participate in the design and conduct of the study, in the collection, analysis, and interpretation of the data, or in the preparation, review, or approval of the manuscript). 


\section{Conflict of interest}

There are no potential conflicts of interest that relate to the manuscript.

\section{References}

[1] Spagnoli LG, Mauriello A, Sangiorgi G, et al. Extracranial thrombotically active carotid plaque as a risk factor for ischemic stroke. JAMA 2004;292:1845-52.

[2] Redgrave JN, Lovett JK, Gallagher PJ, Rothwell PM. Histological assessment of 526 symptomatic carotid plaques in relation to the nature and timing of ischemic symptoms: the Oxford plaque study. Circulation 2006;113: 2320-8.

[3] Virmani R, Ladich ER, Burke AP, Kolodgie FD. Histopathology of carotid atherosclerotic disease. Neurosurgery 2006;59:S219-27, discussion S213.

[4] Golledge J, Greenhalgh RM, Davies AH. The symptomatic carotid plaque. Stroke 2000;31:774-81.

[5] Sacco RL, Adams R, Albers G, et al. Guidelines for prevention of stroke in patients with ischemic stroke or transient ischemic attack: a statement for healthcare professionals from the American Heart Association/American Stroke Association Council on Stroke: co-sponsored by the Council on Cardiovascula Radiology and Intervention: the American Academy of Neurology affirms the value of this guideline. Stroke 2006;37:577-617.

[6] Goldstein LB, Adams R, Alberts MJ, et al. Primary prevention of ischemic stroke: a guideline from the American Heart Association/American Stroke Association Stroke Council: cosponsored by the Atherosclerotic Peripheral Vascular Disease Interdisciplinary Working Group; Cardiovascular Nursing Council; Clinical Cardiology Council; Nutrition, Physical Activity, and Metabolism Council; and the Quality of Care and Outcomes Research Interdisciplinary Working Group: the American Academy of Neurology affirms the value of this guideline. Stroke 2006;37:1583-633.

[7] Takaya N, Yuan C, Chu B, et al. Association between carotid plaque characteristics and subsequent ischemic cerebrovascular events: a prospective assessment with MRI-initial results. Stroke 2006;37:818-23.

[8] Chu B, Ferguson MS, Chen H, et al. Magnetic resonance imaging features of the disruption-prone and the disrupted carotid plaque. JACC Cardiovasc Imag 2009;2:883-96.

[9] Saam T, Cai J, Ma L, et al. Comparison of symptomatic and asymptomatic atherosclerotic carotid plaque features with in vivo MR imaging. Radiology 2006;240:464-72.

[10] Fabiano S, Mancino S, Stefanini M, et al. High-resolution multicontrastweighted MR imaging from human carotid endarterectomy specimens to assess carotid plaque components. Eur Radiol 2008;18:2912-21.

[11] Coli S, Magnoni M, Sangiorgi G, et al. Contrast-enhanced ultrasound imaging of intraplaque neovascularization in carotid arteries: correlation with histology and plaque echogenicity. J Am Coll Cardiol 2008:52:223-30.

[12] Underhill HR, Hatsukami TS, Fayad ZA, Fuster V, Yuan C. MRI of carotid atherosclerosis: clinical implications and future directions. Nat Rev Cardiol 2010;7:165-73.

[13] Falk E, Shah PK, Fuster V. Coronary plaque disruption. Circulation 1995;92:657-71.
[14] Virmani R, Kolodgie FD, Burke AP, Farb A, Schwartz SM. Lessons from sudden coronary death: a comprehensive morphological classification scheme for atherosclerotic lesions. Arterioscler Thromb Vasc Biol 2000;20:1262-75.

[15] Libby P, Ridker PM, Hansson GK. Inflammation in atherosclerosis: from pathophysiology to practice. J Am Coll Cardiol 2009;54:2129-38.

[16] Mauriello A, Sangiorgi GM, Virmani R, et al. A pathobiologic link between risk factors profile and morphological markers of carotid instability. Atherosclerosis 2010;208:572-80.

[17] Wilson PW, D’Agostino RB, Levy D, et al. Prediction of coronary heart disease using risk factor categories. Circulation 1998;97:1837-47.

[18] Grundy SM, Cleeman JI, Daniels SR, et al. Diagnosis and management of the metabolic syndrome: an American Heart Association/National Heart, Lung, and Blood Institute Scientific Statement. Circulation 2005;112:2735-52.

[19] Vergara IA, Norambuena T, Ferrada E, Slater AW, Melo F. StAR: a simple tool for the statistical comparison of ROC curves. BMC Bioinformatics 2008;9:265.

[20] Akobeng AK, Thomas AG. Enteral nutrition for maintenance of remission in Crohn's disease. Cochrane Database Syst Rev 2007 [CD005984].

21] Mantovani A, Sica A, Sozzani S, et al. The chemokine system in diverse forms of macrophage activation and polarization. Trends Immunol 2004;25:677-86.

[22] Stoger JL, Goossens P, de Winther MP. Macrophage heterogeneity: relevance and functional implications in atherosclerosis. Curr Vasc Pharmacol 2010;8:233-48.

23] Fabriek BO, Dijkstra CD, van den Berg TK. The macrophage scavenger receptor CD163. Immunobiology 2005;210:153-60.

[24] Kovanen PT. Mast cells: multipotent local effector cells in atherothrombosis. Immunol Rev 2007;217:105-22.

[25] Moreno PR, Fuster V. New aspects in the pathogenesis of diabetic atherothrombosis. J Am Coll Cardiol 2004:44:2293-300.

[26] Ceriello A, Motz E. Is oxidative stress the pathogenic mechanism underlying insulin resistance, diabetes, and cardiovascular disease? The common soil hypothesis revisited. Arterioscler Thromb Vasc Biol 2004;24:816-23.

[27] Adams MR, Kinlay S, Blake GJ, et al. Atherogenic lipids and endothelial dysfunction: mechanisms in the genesis of ischemic syndromes. Annu Rev Med 2000;51:149-67.

[28] Barter PJ, Nicholls S, Rye KA, Anantharamaiah GM, Navab M, Fogelman AM. Antiinflammatory properties of HDL. Circ Res 2004;95:764-72.

[29] Schiffrin EL. Beyond blood pressure: the endothelium and atherosclerosis progression. Am J Hypertens 2002;15:S115-22.

[30] Spagnoli LG, Mauriello A, Palmieri G, et al. Relationships between risk factors and morphological patterns of human carotid atherosclerotic plaques. A multivariate discriminant analysis. Atherosclerosis 1994;108:39-60.

[31] Li ZY, Howarth SP, Tang T, Gillard JH. How critical is fibrous cap thickness to carotid plaque stability? A flow-plaque interaction model. Stroke 2006;37:1195-9.

[32] Davies PF. Hemodynamic shear stress and the endothelium in cardiovascular pathophysiology. Nat Clin Pract Cardiovasc Med 2009;6:16-26.

[33] Kwee RM. Systematic review on the association between calcification in carotid plaques and clinical ischemic symptoms. J Vasc Surg 2010;51:1015-25.

[34] Wahlgren CM, Zheng W, Shaalan W, Tang J, Bassiouny HS. Human carotid plaque calcification and vulnerability. Relationship between degree of plaque calcification, fibrous cap inflammatory gene expression and symptomatology. Cerebrovasc Dis 2009;27:193-200. 